\title{
Geometric tools for complex interfaces: from lung surfactant to the mussel byssus
}

\author{
Luka Pocivavsek, ${ }^{a}$ Brian Leahy, ${ }^{b}$ Niels Holten-Andersen, ${ }^{a}$ Binhua Lin, $^{b}$ Ka Yee C. Lee ${ }^{a}$ and Enrique Cerda*c
}

DOI: $10.1039 / \mathrm{b} 817513 f$

Interfaces are ubiquitous in nature and absolutely key for life as illustrated by such complex interfaces as the cell membrane and the endothelial and epithelial linings of tissues. The mechanical properties of these interfaces play an important role in their biological functions. In this highlight, we describe our recent work (Pocivavsek et al., Science, 2008, 320, 912) using geometry as a tool for studying the behavior of complex interfaces. General scaling laws emerging from studying the shape of elastic interfaces can in turn be used in their characterization. Interfacial wrinkling is a well known phenomenon, however, the geometric patterns seen at biological interfaces are often far from idealized sinusoidal wrinkles. We show how more complex and non-linear patterns naturally emerge from wrinkles and how material properties can be extracted from these non-linear geometries.

\section{Introduction}

Whether something is hard or soft, stretchy or rigid, pliable or brittle is almost second nature to most people. This knowledge comes from experiences gained through interactions with the world around us. So though most

${ }^{a}$ Department of Chemistry and JFI, University of Chicago, ChicagoIL, 60637, USA

${ }^{b}$ Center for Advanced Radiation Sources and

JFI, University of Chicago, ChicagoIL, 60637, USA

${ }^{c}$ Departamento de Física, Universidad de Santiago, Av. Ecuador 3493, Santiago-Chile. E-mail: enrique.cerda@usach.cl children happily climbing a tree are oblivious to the reasons why a particular branch will hold their weight while another will not, they, through some cuts and bruises, eventually become good judges of this. While such experiential knowledge of material properties is valuable, a quantitative understanding of how materials respond to strains and stresses through theories of elasticity could greatly expand the use of materials and limits to which they can be taken.

Robert Hooke was the first to discuss the elastic property of materials in a general way. In his 1678 paper "de Potentia Restitutiva" Hooke notes: "It is very evident that the Rule or Law of
Nature in every springing body is, that the force or power thereof to restore itself to its natural position is always proportionate to the distance or space it is removed therefrom". ${ }^{1}$ This statement embodies what we know as Hooke's Law, the basis of linear elasticity. The early elasticians were influential not only in their theoretical ideas but also their experimental work. Hooke found that he could compare the springiness or elasticity of different materials (like metal, wood, stones, baked earth, hair, horns, silk, bones, sinews, and glass) by controllably stretching them and creating force versus distance curves. ${ }^{1}$ Thus the quantified stretchability of a material (its



Luka Pocivavsek is a graduate and medical student at the University of Chicago. He is pursuing his PhD in physical chemistry with Prof. Ka Yee Lee. His thesis is focused on elucidating the mechanisms by which model lung surfactant monolayers spread at the airl liquid interface lose mechanical stability when laterally compressed. He has spent a considerable amount of time also at the Universidad de San-

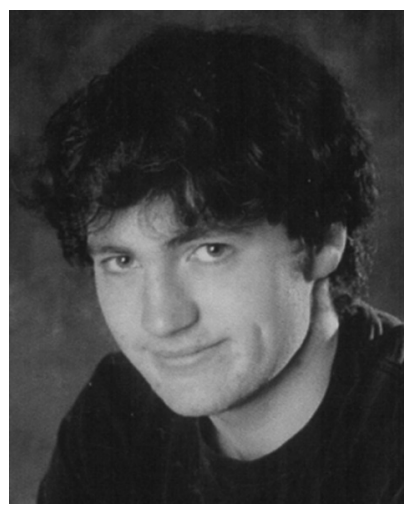

Brian Leahy is a fourth year undergraduate at the University of Chicago, where he is studying physics. He is interested in studying the mechanical response of metal nanoparticle monolayers under stress, as well as how that affects their optical properties. Brian plans on continuing his studies in material science after he completes his degree.

tiago de Chile (USACH) working under Prof. Enrique Cerda on generalizing the type of mechanical instabilities seen in lung surfactant to any elastic interface. 
Young's modulus) became a powerful way of classifying materials.

Since this highlight deals with the problems of defining and measuring elastic properties of complex interfaces, it is useful to note some assumptions that underlie Hooke's approach. ${ }^{\mathbf{1 , 2}}$ First, the elastic nature of a material is considered a bulk thermodynamic property much like its density. And like its density, it is reasonably assumed that the elastic response changes little within some window of perturbations (temperature, pressure, surrounding medium, etc.). In other words, it is assumed that the material is in a stable equilibrium state. Since the material is stable, a controlled and slow deformation of an isolated sample will provide a good characterization of its elastic properties.
As early as the turn of the twentieth century, biologists were applying theories of elasticity based upon this classical approach when probing biological tissues from single cells to whole organs. ${ }^{2}$ Even today, though we have developed very precise measuring tools like atomic force microscopy and optical tweezers to probe single cells and even single molecules, our analysis continues to rely essentially on Hooke. ${ }^{2}$ However, it has become apparent that difficulties are often encountered when trying to characterize biological tissues with standard (Hookean) methods that rely on the above mentioned assumptions of equilibrium.

On the single cell level, for example, the cytoskeleton demonstrates the non-equilibrium nature of biological living materials that needs to be addressed. Work on the cytoskeletal network has shown that in the cell these networks are under a prestrain provided by the constant work of myosin motors, which changes the elastic response of the network significantly., Without the pre-strain, model networks in vitro are much softer than in vivo. However, placing the model network under a pre-load (akin to the work molecular motors do in the cell) stiffens it to the order of a living cell. ${ }^{3,5}$ From a thermodynamic point of view the myosin motors in the cell do work on the actin network powered by the chemical energy stored in ATP. ${ }^{6}$ Since work is constantly done by the molecular motors to keep the actin network in the desired mechanical state, its elasticity is not that of the equilibrium ground state but of the non-equilibrium pre-stressed state. ${ }^{4,7}$

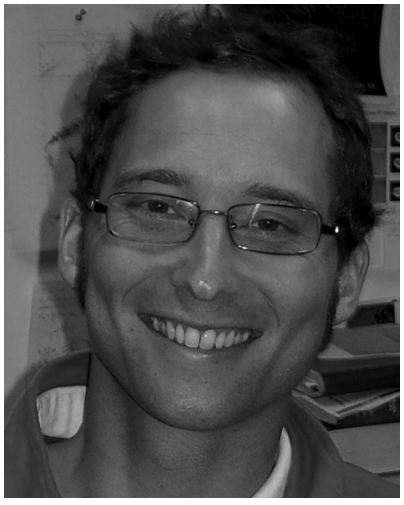

Dr Niels Holten-Andersen received his $P h D$ in the Biomolecular Science and Engineering program at the University of California, Santa Barbara, in 2008, characterizing the protective cuticle of mussel holdfast threads under the guidance of Professor J. Herbert Waite. He is now a postdoctoral researcher in Professor K. Y. C. Lee's group at the Department of Chemistry of the University of Chicago studying the effect of lipophilic proteins and peptides on the mechanical properties of lipid monolayers.



Dr Binhua Lin obtained her PhD in Physics at Northwestern University in 1990. Her thesis research was on the study of structural and phase transitions of Langmuir monolayers of fatty acids using synchrotron X-ray scattering. She is a beamline scientist, in charge of a liquid surfacelinterfacial $X$-ray scattering facility in ChemMatCARS (affiliated to the University of Chicago) at the Advanced Photon Sources, Argonne National Laboratories. Her interests are in structural and dynamic properties of monolayers of metal nanoparticles confined at liquid surfaces/interfaces and the behavior of colloidal suspensions confined in narrow channels.

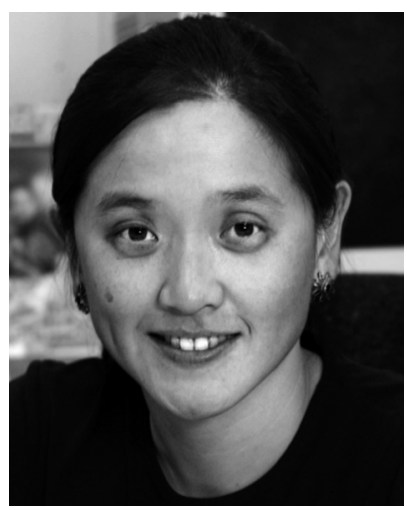

Dr Ka Yee C. Lee received her PhD in Applied Physics at Harvard University in 1992 under the supervision of Eric Mazur. Her thesis was on optical studies of capillary waves at the air-water interface. After her postdoctoral training at Stanford and the University of California at Santa Barbara, she joined the University of Chicago, and is now a Professor of the Department of Chemistry, the Institute for Biophysical Dynamics, and the James Franck Institute. Her research focuses on the interaction of lipids with proteins or polymers at interfaces, and has carried out biophysical studies to elucidate the functioning of lung surfactant.

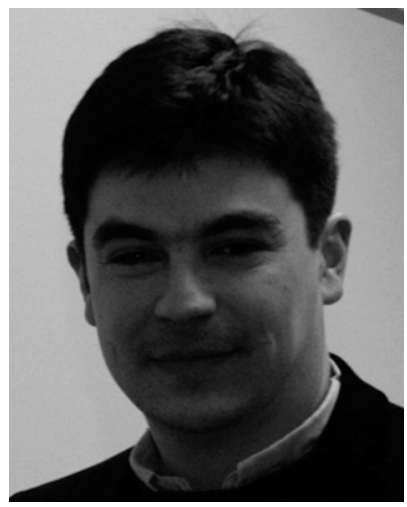

Dr Enrique Cerda obtained his PhD in Physics at Universidad de Chile in 1996 under the supervision of Professor Enrique Tirapegui. After his postdoctoral work with Professor L. Mahadevan at MIT on crumpling of thin films he joined the Universidad de Santiago de Chile. His research focuses on the physics and geometry of thin surfaces and the theoretical and experimental study of the crumpling, folding, wrinkling and fracture of soft and stiff elastic surfaces. 
The problems with a classical Hookean approach become even more severe when the system under study is not a bulk material but an interface. By an interface, we mean a physical region that exists between and separates two regions of bulk space. Nature abounds with interfaces of all types. One of the simplest is the air/water interface where a layer of water molecules at the surface interacts with bulk water on one side and air on the other. In the lungs, such an air/water interface is made more complex by the placement of a thin layer of lipids and proteins (lung surfactant) at the surface; in this case, the interface is no longer only a layer of water molecules but the layer of larger and chemically more intricate lipids and proteins which now separate the bulk water from the bulk air.

Interfaces need not be limited to molecular scales. For example our skin is composed of specialized epithelial cells that act as an interface separating the outside air from the muscle and tissue lying underneath it; in muscular arteries the smooth muscle of the vessel wall is separated from the blood inside the vessel lumen by a monolayer of specialized endothelial cells and a network of collagen and elastin known as the basement membrane. In these examples, the interface is microns thick and composed of materials that are intricately more complex in their chemical structure and organization than the nanometre thin water or the lipid-protein molecular layers described above.

Despite the large separation in length scales and chemical composition, these interfaces are united by their geometry. If the system is such that the thickness of the interfacial layer becomes small relative to the thickness of the bulk layers it separates, then the interface mathematically and physically can be treated as a membrane. Therefore, the different systems mentioned above while compositionally very different can all be treated mathematically as a two-dimensional entity embedding in three-dimensional space.

All but the simplest interfaces mentioned above are in a non-equilibrium state which makes probing their material properties via a Hookean approach unsuitable. Their properties can nonetheless be extracted by exploiting the unifying geometry of interfaces as membranes. Based on the observation and study of the geometry of such interfaces as they are stressed, a new trend of research exploring indirect methods to obtain the material properties of thin films through their two-dimensional geometry is developing. ${ }^{8}$ Smooth sinusoidal undulations (wrinkles) observed at an interface under different applied loads $^{9-12}$ or the tear shapes left upon fracture of thin adhesive films upon pealing, ${ }^{13}$ have been shown to hold valuable information about the stiffness, adhesion, and fracture energy of thin membranes.

We have recently developed a general model for the evolution of elastic interfaces, and below we present a new pattern, that we define as folds, from which the material properties of an interface far from thermodynamic equilibrium can be obtained. ${ }^{14}$ Our method is non-traditional in the sense that material constants like bending stiffness and Young's modulus are extracted from geometric scaling laws instead of force $v s$. distance curves. Moreover, our methods can be applied to studies of mechanical properties of living tissues in their in vivo state.

\section{Wrinkle to fold transition}

The study of wrinkled surfaces has become an interesting subject of research in past years. ${ }^{10}$ Wrinkling is observed in everyday length scales, in our clothing and skin, at small length scales in nanotubes, cell membranes and biological tissues, and also at large scales of geological size. This ever presence of wrinkling is a testament to the ubiquitous existence of elastic interfaces in nature. We concentrated our studies on the elastic stability of interfaces. In particular, we looked at membranes resting on softer substrates. A piece of polyester microns thick resting on either a water surface or a soft gel was our toy model. Laterally compressing such a membrane gives rise to a periodic wrinkling pattern (see Fig. 1a), small amplitude periodic undulations of the membrane. We showed that they appear as the first order linear solution to the interfacial energy. However, the wrinkled state can be suppressed by yet another state where membrane energy becomes sharply focused in local regions with no periodic distribution, a state we term the fold state.

In the simplest form our supported membrane can be described by two energies: the elastic energy of the membrane and the energy of the substrate on which it rests. The elastic energy of a membrane contains a stretching term that represents the cost of increasing or decreasing the membrane area, and a bending term that embodies the cost of distorting the flat membrane into some non-flat geometry. The membrane stretching energy can be dropped due to the large mismatch in the energy needed to stretch versus to bend a membrane. A real world illustration of this is a simple piece of paper. Try pulling on a piece of paper to increase its area and it becomes quickly apparent that its resistance to stretching is enormous.

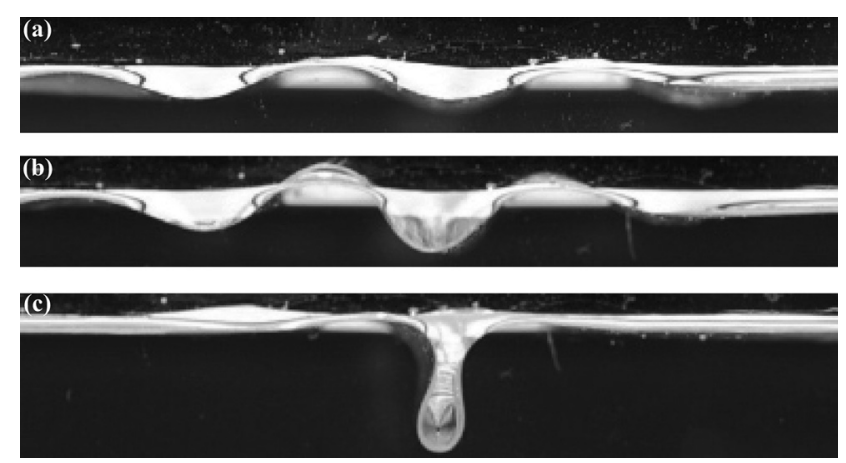

Fig. 1 Polyester film on water transitioning from an extended wrinkled state to a localized folded state. The polyester film is $h \sim 10 \mu \mathrm{m}$ thick and clamped at the boundaries. The macroscopic system is imaged from the side allowing for careful determination of amplitudes and wavelengths as well as a clear view of the wrinkle to fold transition. (a) Wrinkled state at low confinement with a wavelength $\lambda \sim 1.6 \mathrm{~cm}$. (b) Upon further compression the wrinkles increase their amplitude, but the one in the middle grows more than its neighbors. (c) The wrinkles collapse into one fold in the middle where all the excess of length is stored. The film becomes flat except for this localized defect. 
Bending the same piece of paper is trivial. Since resistance to membrane stretching is linearly proportional to membrane thickness ( $h$ ) and membrane bending goes as thickness cubed $\left(h^{3}\right)$, the ease of bending versus stretching changes quadratically with thickness. ${ }^{\mathbf{8}, 15}$

The substrate energy comes from the potential energy incurred due to the displacement of the substrate underneath as the membrane changes shape from some flat geometry to a wrinkled or folded one. Since the substrate is a bulk material, the reverse arguments of those for the membrane apply. Only stretching terms of the substrate potential energy need to be considered and bending terms can be dropped.

Mathematically we can describe such an interfacial membrane with an energy of the form

$$
\begin{gathered}
U=B \times(\text { Membrane Bending })+ \\
K \times(\text { Substrate Stretching })
\end{gathered}
$$

The first term is the bending energy where $B$ is the bending stiffness of the membrane. The second term is the substrate potential with $K$ being the substrate stiffness.

If the membrane is compressed only slightly the problem can be solved exactly and one obtains periodic wrinkles as the minimal solutions. The wavelength of the wrinkles is set by a balance of the bending energy and the substrate potential, and is given by $\lambda \sim(B / K)^{1 / 4}$. There are alternative ways of deriving this wavelength, ${ }^{9,16,17}$ which strengthens the conclusion that $\lambda$ is the essential length scale describing the energy of a slightly compressed membrane.

Understanding $\lambda$ has significant experimental consequences. If we observe an interface that is wrinkled and it is reasonable to assume the interface to be under compression and treatable as an elastic membrane, then measuring the wrinkle wavelength can provide information about the in-plane strength of the membrane and the stiffness of the substrate. The bending stiffness $(B)$ can be approximated at $E \times h^{3}$, where $E$ is the Young's modulus, ${ }^{18,19}$ so measuring $h$ and $B$ can give information about the strength of intermolecular interactions in the membrane. Similarly, provided that alternative approximations from other experiments or theory exist for the bending stiffness, the strength of the substrate, $K$, can also be extracted.

The crux of the wrinkling problem is that wrinkled surfaces can become unstable. The instability manifests itself as a focusing phenomenon: compression of a wrinkled membrane leads to a distributed growth of wrinkle amplitudes until a critical compression is reached. At that point, the amplitudes of one or a few wrinkles grow while that of the rest decay to zero. This transition is what we have termed the wrinkle-to-fold transition.

In our analysis, we were able to define a critical compression $\left(\Delta_{c}\right)$ for the beginning of the wrinkle-to-fold transition for membranes on very soft substrates like liquids. In such cases, when substrate resistance to shearing is minimal and the primary substrate potential is gravitational, i.e. $K=\rho g$, a wrinkled surface begins to lose stability once it has been compressed by a third of its initial wavelength $\left(\Delta_{c}=\lambda / 3\right)$. The ten micron thick polyester membrane on water in Fig. 1 for example is approximately $10 \mathrm{~cm}$ in length and has wrinkles with a wavelength of $1.5 \mathrm{~cm}$ (Fig. 1a). Further compressing this surface until a confinement of $0.5 \mathrm{~cm}$ is reached, the onset of folding is observed (Fig. 1b). The transition is complete once all the excess membrane length present in the wrinkles is redirected and focused into the fold (see Fig. 1c), which occurs when the membrane has been laterally compressed by a full wavelength $(\Delta=\lambda)$. In other words, once the membrane is compressed by $1.5 \mathrm{~cm}$ in the above example, no wrinkles remain and all the excess length is focused into the one fold seen in Fig. 1c.

The interesting question from an experimental view is whether the size of the folds for a purely folded membrane can be used to extract some useful interfacial material parameters as with a wrinkled membrane. Obtaining scaling laws for the above energy in the fold regime, we have determined that the fold size should scale as $\ell \sim\left(\frac{B}{K}\right)^{1 / 2} \frac{1}{\Delta}$. However, using the scaling law for $\lambda$ this can be rewritten as $\ell$ $\sim \lambda^{2} / \Delta$. Since $\Delta \approx \lambda$ toward the end of the transition, we can conclude that the fold size should be proportional to the initial wrinkle wavelength, from which parameters like $B$ and $K$ can be extracted.
These ideas are not limited to macroscopic films. The wrinkle-to-fold transition is also observed using nanometre thin membranes. ${ }^{20}$ Fig. $2 \mathrm{a}$ and $2 \mathrm{~b}$ show gold nanoparticles $5 \mathrm{~nm}$ in diameter at an air/glycerol interface compressed to form a self-assembled trilayered film $15 \mathrm{~nm}$ thick. Using light microscopy one can observe the initial periodic wrinkles with $\lambda \sim 10 \mu \mathrm{m}$ (Fig. 2a). If the compression is stopped, the surface remains wrinkled. However, further confinement leads to the focusing behavior observed in the macroscopic polyester film. Fig. 2b shows the appearance of a pattern of localized folds taking the place of the wrinkles.

The usefulness of our conclusions is that even if a perfectly wrinkled surface does not exist, the material properties of the interface can be extracted from the fold geometry. Many biologically interesting interfaces behave as elastic membranes resting on a liquid or a very soft gel-like substrate. One example is the thin monolayer of lipids and proteins covering our lungs. Lung surfactant is continuously compressed and expanded during the breathing cycle, and its response to lateral compaction, i.e. its mechanical stability, has been shown to be key for its biological functioning. ${ }^{21,22} \mathrm{~A}$ properly functioning lung surfactant monolayer must respond to compaction by folding. ${ }^{23}$ In vitro microscopy studies of model surfactants at the air/water interface shows fold amplitudes of a few microns (Fig. 2d) yet have failed to capture wrinkles (Fig. 2c). ${ }^{1424-26}$ However, using our derived scaling $\ell \approx \lambda$ and assuming the substrate potential to be gravitational, we can predict the surfactant monolayer to have a bending stiffness on the order of $10 \mathrm{kT}$, which is in agreement with previous work. ${ }^{7,17}$

While the examples presented thus far all deal with a thin elastic membrane sitting on a fluid, a fluid substrate, however, is not necessary for the wrinkleto-fold transition. Fig. 3a shows a laterally compressed macroscopic polyester film adhered to a soft gel. Smooth wrinkling (Fig. 3a inset) becomes unstable, eventually localizing into several folds and at the same time relaxing the rest of the interface (Fig. 3a). Thus, this focusing effect and the wrinkle-to-fold transition should generally occur when the thin membrane and the substrate foundation are significantly mismatched in their 

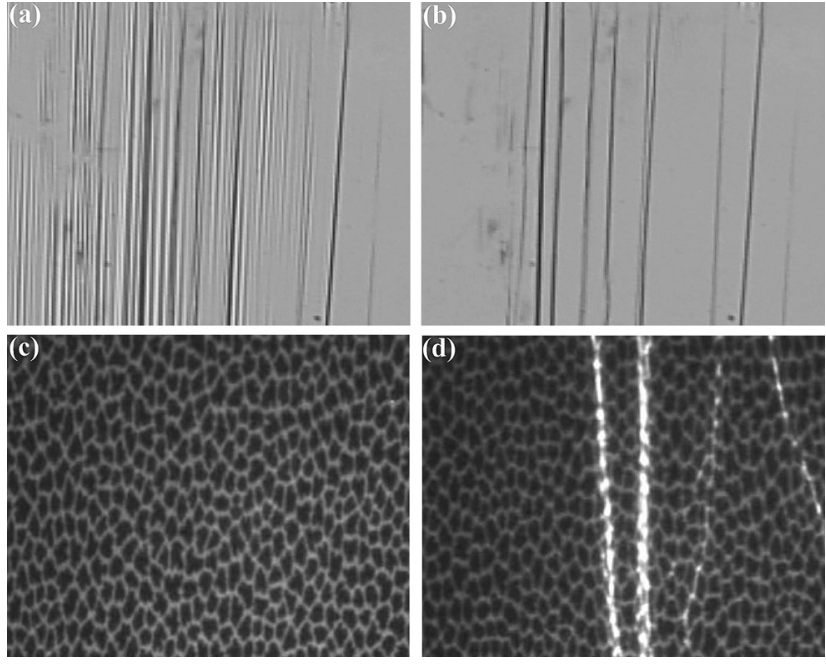

Fig. 2 (a) Bright field microscopy images of a trilayer of colloidal gold nanoparticles on a glycerol/ water surface held together by van der Waals forces ${ }^{20}$ showing a wrinkled surface when slightly compressed. Here the layer has $h \sim 15 \mathrm{~nm}$ (as determined by AFM) and the wrinkles observed have a wavelength $\lambda \sim 10 \mu \mathrm{m}$. (b) The same layer at further compression has the wrinkles collapse into a pattern of localized folds. (c) and (d) show fluorescence images (750 microns across) of a model lung surfactant system $(h \sim 2 \mathrm{~nm})$ composed of a $7: 3$, mol : mol mixture of 1,2-dipalmitoylsn-glycero-3-phosphocholine (DPPC) and 1-palmitoyl-2-oleoyl-sn-glycero-3-[phospho-rac-(1-glycerol)] (POPG $)^{25}$ at an air/water interface. Even at high compression, low-amplitude wrinkles are not observed (c) (likely due to the poor scattering of the mostly hydrocarbon lipids). However, folds (d) (appearing as bright lines running perpendicular to the direction of compression) are easily visualized with fluorescence due to the high density of surface lipids and dye pulled into a given fold. The amount of material pulled into a given fold has been previously carefully measured. ${ }^{26} \mathrm{We}$ used the size of the folds and our scaling law $\lambda \sim \ell$ to extract out the bending stiffness of the lung surfactant monolayer to be on the order of $10 \mathrm{kT}$ in agreement with previous work. ${ }^{7,17}$
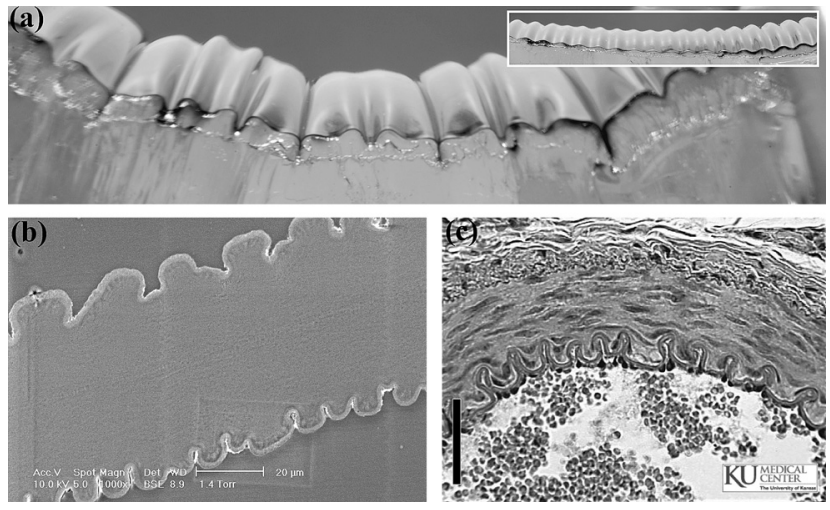

Fig. 3 (a) Highly compressed polyester film on top of a gel substrate (image is $4 \mathrm{~cm}$ across). The excess of length at high compressions is localized into multiple folds, giving the surface a convoluted shape. Compare this structure with the regular sinusoidal wrinkles (see inset) observed for moderate compressions. (b) Cross-sectional scanning electron microscopy images of thread filament used by mussel shellfish to adhere to rocks. ${ }^{29}$ Prior work has shown that the fiber is composed of a softer collagenous core covered by a harder protective cuticle. ${ }^{29,30}$ (c) A histological slide of a muscular artery cross-section ( $\mathrm{bar}=50 \mu \mathrm{m}^{33}$ ), the luminal (lower part in image) wall of the artery is covered in fold undulations. We conjecture that the folding layer is composed of the cellular endothelium as well as the layer of elastic biopolymers to which the endothelial cells are attached (basement membrane). The substrate here is the layer of arterial smooth muscle cells (top part of image).

elastic properties. In the case of a fluid substrate, the transition occurs as described. In the case of soft gels, such as the system in Fig. 3a, where the ratio of the Young's moduli of the membrane $\left(E_{\mathrm{m}}\right)$ and the substrate $\left(E_{\mathrm{s}}\right)$ is approximately one-thousand, localization would still occur but is distributed into several folds. Work on very stiff substrates $\left(E_{\mathrm{m}} / E_{\mathrm{s}}<100\right)$ has shown the persistence of wrinkles at large confinement with no stress focusing. ${ }^{27,28} \mathrm{We}$ believe the relaxation of wrinkles into multiple folds is linked to the underlying ability of the substrate to stretch and shear. We predict that in the decreasing range $1000>E_{\mathrm{m}} / E_{\mathrm{s}}$ $>100$, the number of wrinkles relaxing into one fold should be lower, giving rise to a greater density of folds.

The presence of folds and the fold density both in-themselves hold valuable information about interfacial material properties. To highlight the application of these conclusions we present two biological examples. Fig. 3b shows a longitudinal cut through one of the hold fast threads of the mussel shellfish, collectively called the byssus. ${ }^{29}$ The shellfish uses the byssus to adhere itself to rocks and the ocean floor, making it indispensable to its survival. ${ }^{29}$ In Fig. 3b, the external surface of the thread is convoluted into folds. Given the presence of folds and their density at the surface, we predict based upon these geometric observations that the thread surface is composed of a membrane whose thickness is a fraction of the thread diameter, yet whose elastic stiffness is greater than the thread core. Furthermore, given the density of folds as compared to Fig. 3a, we predict the ratio of stiffness to be in the range $1000>E_{\mathrm{m}} / E_{\mathrm{s}}$ $>100$. This is in agreement with independent experiments characterizing the material properties of the byssus and showing a mismatch in stiffnesses close to $100 .^{29,30}$

A second example highlighting the power of geometric analysis for complex interfaces comes from within us. Fig. 3c shows a cross-section through a medium muscular artery. The inner arterial wall (luminal side in contact with blood) shows beautiful undulations whose shape is again similar to the folds seen in Fig. 3a. By deduction, we hypothesize using only the interfacial geometry that a stiffness gradient should exist across the arterial wall, with the luminal layer being stiffer and the outer layer being somewhat softer. Based on fold density as compared to Fig. 3b, the mismatch in stiffnesses should also be close to 100. Our predictions are again validated by experiments. ${ }^{31,32}$ Anatomically, the stiffer luminal layer is an interface composed of endothelial cells and biopolymers (the 
basement membrane) that separate the softer outer muscle layer of the artery from the blood.

In the case of the byssus and the artery, other methods have been used to validate our predictions. However, since our methods are purely observational, relying on analysis of the geometry of an interface, they could be extended to systems that can not be probed by traditional material characterization. Candidates include biological tissues where in vivo imaging provides a glimpse of the system in its functional non-equilibrium state. Interfaces as compositionally diverse as a piece of polyester and a nanometre thin layer of surfactant on water or the far more intricate biological interfaces of the byssus and artery all show similar behavior. This universal response of elastic interfaces emerges because their physics is dominated by their membranelike geometry and provides a potentially powerful tool in material sciences.

\section{Acknowledgements}

We thank Tom Witten for many fruitful discussions as well as his leadership of the NSF Inter-American Materials Collaboration: Chicago-Chile (DMR-0303072) under whose support this collaboration started. We likewise thank Mati Meron for many rich discussions and Stuart Rice, Francisco Melo, Jorge Pavez, Andrej Pocivavsek, and Kinlok Lam for experimental help. This work was supported in part by the University of Chicago MRSEC program of the NSF (DMR0213745) and the US-Israel Binational Foundation (2006076). LP thanks the University of Chicago MSTP for support (NIGMS/MSNRSA 5T32GM07281). NHA thanks the Danish Natural Science Research Council for a Post-Doctoral Fellowship (No. 272-08-0087). KYCL is grateful for support from March of Dimes (No. 6-FY07-357). BL and BL acknowledge the support of NSF/DOE grant No. CHE-0535644 for ChemMatCARS. EC acknowledges the support of Anillo No ACT 15, FONDAP Grant No. 11980002, and Fondecyt Project No. 1050083.

\section{References}

1 S. P. Timoshenko, History of Strength of Materials, Dover, Mineola, New York, 1983, pp. $19-20$.

2 A. E. Pelling and M. A. Horton, An Historical Perspective on Cell Mechanics, Pflugers Arch. - Eur. J. Physiol., 2008, 456, 3.

3 K. E. Kasza, A. C. Rowat, J. Liu, T. E. Angelini, C. P. Brangwynne, G. H. Koenderink and D. A. Weitz, The Cell as a Material, Current Opinion in Cell Biology, 2007, 19, 101.

4 D. Mizuno, C. Tardin, C. F. Schmidt and F. C. MacKintosh, Nonequilibrium Mechanics of Active Cytoskeletal Networks, Science, 2007, 315, 370.

5 M. L. Gardel, F. Nakamura, J. H. Hartwig, J. C. Crocker, T. P. Stossel and D. A. Weitz, Prestressed F-actin networks cross-linked by hinged filamins replicate mechanical properties of cells, Proc. Natl. Acad. Sci. U. S. A., 2006, 103, 1762.

6 B. Alberts, D. Bray, J. Lewis, M. Raff, K. Roberts, and J. D. Watson, Molecular Biology of the Cell, Garland Publishing, New York, 1994.

7 D. Boal, Mechanics of the Cell, Cambridge University Press, Cambridge, 2002.

8 T. A. Witten, Stress focusing in elastic sheets, Reviews of Modern Physics, 2007, 79, 643 .

9 E. Cerda and L. Mahadevan, Geometry and physics of Wrinkling, Phys. Rev. Lett., 2003, 90, 074302.

$10 \mathrm{~J}$. Genzer and J. Groenewold, Soft matter with hard skin: From skin wrinkles to templating and material characterization, Soft Matter, 2006, 2, 310.

$11 \mathrm{C}$. Stafford et al., A buckling-based metrology for measuring the elastic moduli of polymeric thin films, Nature Materials, 2004, 3, 545.

$12 \mathrm{~J}$. Huang et al., Capillary wrinkling of floating thin polymer films, Science, 2007, 317, 650 .

13 E. Hamm, P. Reis, M. LeBlanc, B. Roman and E. Cerda, Tearing as a test for mechanical characterization of thin adhesive films, Nat. Mater., 2008, 7, 386.

14 L. Pocivavsek, R. Dellsy, A. Kern, S. Johnson, B. Lin, K. Y. Lee and E. Cerda, Stress and Fold Localization in Thin Elastic Membranes, Science, 2008, 320, 912 .

15 J. W. S. Rayleigh, Theory of Sound Dover, New York, 1922, vol. 1, (ch. Xa), pp. 19-20.

16 Q. Zhang and T. A. Witten, Microscopic wrinkles on supported surfactant monolayers, Phys. Rev. E, 2007, 76, 041608.

17 S. T. Millner, J. F. Joanny and P. Pincus, Buckling of Langmuir Monolayers, Europhys. Lett., 1989, 9, 495.

18 S. P. Timoshenkoand S. WoinowskyKreiger, Theory of Plates and Shells, McGraw-Hill, 2nd edn, 1964.

19 L. D. Landau and E. M. Lifshitz, Theory of Elasticity, Pergamon, NY, 3rd edn, 1986.
20 D. G. Schultz, X. M. Lin, L. Dongxu, J. Gebhardt, M. Meron, P. J. Viccaro and B. Lin, Structure, Wrinkling, and Reversibility of Langmuir Monolayers of Gold Nanoparticles, J. Phys. Chem. B, 2006, 110, 24522.

21 J. A. Zasadzinski, J. Ding, H. E. Warriner, F. Bringezu and A. J. Waring, Current Opinion in Colloid \& Interface Science, 2001, 6, 506.

22 N. J. Miller, C. B. Daniels, S. Schurch, W. M. Schoel and S. Orgeig, The Surface Activity of Pulmonary Surfactant from Diving Mammals, Resp. Phys. Neurobio., 2006, 150, 220.

23 S. Schurch, F. H. Y. Green and H. Bachofen, Formation and structure of surface films: captive bubble surfactometry, Biochimicaet Biophysica Acta, 1998, 1408, 180.

24 L. Pocivavsek, S. L. Frey, K. Krishan, K. Gavrilov, P. Ruchala, A. J. Waring, F. J. Walther, M. Dennin, T. A. Witten and K. Y. C. Lee, Lateral stress relaxation and collapse in lipid monolayers, Soft Matter, 2008, 4, 2019.

25 A. Gopal and K. Y. C. Lee, Morphology and Collapse Transitions in Binary Phospholipid Monolayers, J. Phys. Chem. $B, 2001,105,10348$.

26 Gopal, V. A. Belyi, H. Diamant, T. A. Witten and K. Y. C. Lee, Microscopic folds and macroscopic jerks in compressed lipid monolayers, J. Phys. Chem. B, 2006, 110, 10220.

27 K. Efimenko, M. Rackaitis, E. Manias, A. Vaziri, L. Mahadevan and J. Genzer, Nested self-similar wrinkling patterns in skins, Nat. Mat., 2005, 4, 293.

28 E. Sultan and A. Boudaud, The buckling of a swollen thin gel layer bound to a compliant substrate, J. Applied Mech. in press.

29 N. Holten-Andersen, G. E. Fantner, S. Hohlbauch, J. H. Waite and F. W. Zok, Protective Coatings on Extensible Biofibers, Nat. Mat., 2007, 6, 669.

30 J. M. Lucas, E. Vaccaro and J. H. Waite, A molecular, morphometric and mechanical comparison of the structural elements of byssus from Mytilus edulis and Mytilus galloprovincialis, J. Exp. Bio., 2002, 205, 1807.

31 K. Nagayama, Y. Nagano, M. Sato and T. Matsumoto, Effect of actin filament distribution on tensile properties of smooth muscle cells obtained from rat thoracic aortas, J. Biomech., 2006, 39, 293.

32 J. Candiello, M. Balasubramani, E. M. Schreiber, G. J. Cole, U. Mayer, W. Halfter and H. Lin, Biomechanical properties of native basement membranes, FEBS Journal., 2007, 274, 2897.

$33 \mathrm{M}$. WolfM. Scarbrough, The JayDoc HistoWeb, http://kumc.edu/instruction/ medicine/anatomy/histoweb/vascular/small/ Vasc10s.JPG, The University of Kansas Medical Center. 